\title{
Sec22b regulates inflammatory responses by controlling the nuclear translocation of NF- $\mathrm{KB}$
}

\author{
Guillermo ARANGO DUQUE1‡, Renaud DION1¥, Aymeric FABIÉ1, Julien DESCOTEAUX¹, Simona \\ STÄGER ${ }^{1}$ and Albert DESCOTEAUX ${ }^{*}{ }^{*}$
}

1 INRS - Centre Armand-Frappier Santé Biotechnologie, Université du Québec, Laval, QC, H7V 1B7, Canada

₹ These authors have contributed equally to this manuscript

${ }^{*}$ Corresponding author: Albert Descoteaux

E-mail: albert.descoteaux@inrs.ca

Running title: Sec22b mediates NF- $\kappa$ B translocation

Keywords: Cytokine, intracellular trafficking, nuclear translocation, soluble NSF attachment protein receptor (SNARE), NF-kappa B (NF-KB) 


\begin{abstract}
Soluble NSF attachment receptor (SNARE) proteins regulate the vesicle transport machinery in phagocytic cells. Within the secretory pathway, Sec22b is an ER-Golgi intermediate compartment (ERGIC)-resident SNARE that controls phagosome maturation and function in macrophages and dendritic cells. The secretory pathway controls the release of cytokines and may also impact the secretion of nitric oxide (NO), which is synthesized by the Golgi-active inducible nitric oxide synthase (iNOS). Whether ERGIC SNARE Sec22b controls NO and cytokine secretion, is unknown. Using bone marrow-derived dendritic cells (BMDC), we demonstrated that iNOS colocalizes with ERGIC/Golgi markers, notably Sec22b and its partner syntaxin-5 (Stx5), in the cytoplasm and at the phagosome. Pharmacological blockade of the secretory pathway hindered NO and cytokine release, and inhibited NF- $\kappa B$ translocation to the nucleus. Importantly, RNAi-mediated silencing of Sec22b revealed that $\mathrm{NO}$ and cytokine production were abrogated at the protein and mRNA levels. This correlated with deregulated mitogen-activated protein kinase signalling and reduced nuclear translocation of NF- $\kappa B$. We also found that Sec22b co-occurs with NF- $\kappa B$ in both the cytoplasm and nucleus, pointing to a role for this SNARE in the shuttling of NF- $\kappa$ B. Collectively, our data unveiled a novel function for the ER-Golgi, and its resident SNARE Sec $22 \mathrm{~b}$, in the production and release of inflammatory mediators.
\end{abstract}

\section{Introduction}

The innate immune function of professional phagocytes is channeled through their capacity to ingest and degrade foreign particles and microbes (1). Following particle binding and uptake, phagocytes release a series of small molecules such as nitric oxide (NO) and cytokines that dictate the nature and intensity of the immune response. Particularly in dendritic cells, the phagosome also serves as a platform for antigen processing and crosspresentation (2). The aforementioned processes exert a membrane demand that is actively supplied by endosomes and the secretory pathway, a dynamic group of organelles through which proteins and lipids are synthesized, processed and transported $(3,4)$. While recycling/early endosomes and the endoplasmic reticulum (ER) supply membrane and protein effectors to nascent phagosomes $(5,6)$, the latter also serve as cytokine secretion sites (7). Through sequential membrane exchanges with organelles such as the lysosome, maturing phagosomes acidify and become highly degradative.

Interorganellar vesicular transport is regulated by proteins that reside and determine cargo selectivity and vesicle destination (3). Vesicle docking and fusion rely on organelle-specific members of the soluble $N$-ethylmaleimidesensitive factor attachment protein receptor (SNARE) family $(4,8)$. For instance, the syntaxin (Stx) 4/SNAP23/VAMP3 complex facilitates the passage of TNF from recycling endosomes to the cell membrane $(7,9)$. On the other hand, trafficking within the ER, ER-Golgi intermediate compartment (ERGIC), Golgi apparatus and phagosome circuitry is orchestrated by SNARE complexes involving Sec22b, Stx5 and Stx4 $(10,11)$. Notably, their absence hinders phagosome maturation and antigen crosspresentation $(10,11)$, which translates into phenotypes such as impaired bacterial and tumor clearance (12-14). Moreover, a panmouse knockout of Sec22b is embryonically lethal (15), highlighting the key importance of this ER/ERGIC-resident SNARE in immunity and development.

The inducible nitric oxide synthase 2 (iNOS) bridges homeostatic regulation and immunity via the production of NO from L-arginine (16). NO modulates inflammation, vasodilation and neurotransmission. Importantly, this reactive molecule contributes to the generation of highly microbicidal reactive nitrogen species (RNS) within the phagosome $(17,18)$ that kill internalized bacteria and Leishmania parasites (19). iNOS expression is mainly regulated at the transcriptional level by nuclear factor kappa B (NF- $\kappa \mathrm{B})$, and enzymatic activity is regulated at the translational and post-translational levels. Indeed, iNOS has to assemble into homodimers in order to transform L-arginine into RNS (20). Although its intracellular trafficking remains uncharacterized, iNOS-positive vesicles have 
been observed to reside in ER-associated aggresomes and the trans-Golgi (21), as well as the phagosome surface (17). Therefore, the question emerges as to whether secretory pathway-resident SNAREs regulate iNOS trafficking and function. We showed that iNOS is mainly localized to the ERGIC/Golgi both in the cytoplasm and at the phagosome. Notably, Sec22b knockdown hindered NF- $\mathrm{BB}$ nuclear translocation, thereby decreasing iNOS and cytokine production. We also observed that Sec22b and NF- $\kappa \mathrm{B}$ co-occur in the cytoplasm and the nucleus. These findings highlight a new role for this SNARE in the control of inflammatory responses.

\section{Results \\ iNOS localizes within the secretory pathway in BMDC and is recruited to the phagosome}

In BMDC, the release of NO is induced by both soluble and particulate stimuli (Figure 1A). To elucidate the underlying mechanisms and pathways, we first assessed the localization of iNOS in LPS-stimulated BMDC, using immunofluorescence confocal microscopy. Figure 1B shows that iNOS was present in vesicular structures situated in the cytoplasm and plasmalemma, consistent with its role in NO secretion to the extracellular milieu (16). To further determine the subcellular localization of iNOS in BMDC, we performed co-localization analyses by immunofluorescence confocal microscopy using markers of the ER (CNX, PDI), the ERGIC (ERGIC53, Sec22b, Stx5) the Golgi apparatus (P115) and lipid rafts (CTX) (Figure 1C, D and Figure S1). These analyses revealed that iNOS was widely present in secretory pathway organelles, though most saliently in the ERGIC and the Golgi (Figure 1C, D). Moreover, following phagocytosis of zymosan particles, we observed that iNOS colocalizes with Sec22b, Stx5, and LAMP1 on phagosomes (Figure 2A, B). Collectively, these data suggest a role for the secretory pathway in NO secretion.

\section{The secretory pathway is required for NO and cytokine production}

The ER and the Golgi are critical for the biogenesis and secretion of proinflammatory cytokines such as TNF and IL-6 (4). Here, we assessed the potential role of ER-Golgi trafficking in the control of iNOS activity and NO release by BMDC, and we included TNF and IL-6 in our analysis since their release is mediated by the secretory pathway (4). To this end, we treated BMDC with DMSO or with inhibitors of ER-Golgi trafficking, namely brefeldin A, monensin, and retro-2 (22-24), prior to stimulation with LPS. While monensin and brefeldin A inhibited NO, TNF and IL-6 release, retro-2 only inhibited TNF release, highlighting individual differences in the intracellular trafficking of those molecules (Figure $3 \mathrm{~A}-\mathrm{C}$ ). Of note, both monensin and brefeldin A inhibited iNOS protein expression (Figure 3D-E), suggesting that ER-Golgi trafficking is part of the pathway leading to iNOS expression. To understand how perturbation of the secretory pathway led to the inhibition of NO and cytokine release, we first assessed the impact of monensin on the integrity of LPS-induced signal cascades associated to the expression of iNOS, TNF, and IL-6 $(25,26)$. As shown in Figure 4A, pretreatment with monensin had no effect on LPS-induced phosphorylation of $\mathrm{p} 44 / \mathrm{p} 42$ MAPK (ERK1/2) and degradation of IкB $\alpha$, but impaired the phosphorylation kinetics of p38 and JNK. One of the key downstream event of inducible $I \kappa B \alpha$ degradation is the nuclear translocation of the transcription factor NF- $\kappa \mathrm{B}$, which mediates the expression of a large number of LPS-inducible genes including iNOS, TNF, and IL-6 $(25,26)$. As shown in Figure 4B-C, pretreatment of BMDC with monensin impaired LPS-induced nuclear translocation of NF- $\kappa B$. Collectively, these findings are consistent with the notion that the integrity of the secretory pathway is necessary for optimal NF- $\kappa \mathrm{B}-$ mediated LPS responses in BMDC.

\section{The secretory pathway SNARE Sec22b regulates LPS-induced responses}

The SNARE Sec22b plays a role in ER-Golgi trafficking (27) and in the unconventional secretion of IL-1 $\beta$ in cells treated with autophagy inducers, a process known as secretory autophagy (28). To assess the potential role of Sec22b in the release of NO, we stimulated with LPS DC-like JAWS-II cells transduced with an shRNA to Sec22b (shSec22) or a scrambled (shScr) sequence (10). As shown in Figure 5A-C, Sec22b knockdown 
diminished the release of NO, as well as of TNF and IL-6. To confirm the role of Sec22b in NO secretion and to test whether this mechanism operates in macrophages, we stimulated with LPS RAW264.7 macrophages pretreated with siRNA to Sec22b, its binding partner Stx5, or both. In that regard, knockdown of one or both SNAREs resulted in the inhibition of NO release (Figure 5D). Similar to the effect of pharmacological inhibitors on iNOS expression, Sec22b knockdown diminished iNOS protein levels (Figure 5E), as well as Inos, Tnf and Il6 expression, as assessed by quantitative reverse transcription PCR (RT-qPCR) (Figure 5F).

To ensure that the function of Sec22b on inflammatory mediator production is not contingent on LPS stimulation, we treated Sec22b-KD cells with agonists to TLR2 (peptidoglycan [PGN]), dectin-1/TLR2 (zymosan), TLR2 (L. major promastigotes), TLR7 (imiquimod) or TLR9 (CpG). Twenty-four hours post-stimulation, silencing Sec22b abrogated the induction of NO, TNF and IL- 6 by all of the tested ligands (Figure 6A-C). Because pharmacological blockade of the secretory pathway abrogated inflammatory mediator release (Figure 5), it is possible that the resident SNARE Sec22b is responsible for this abrogation. To test this, we treated shScr and shSec $22 \mathrm{~b}$ cells with brefeldin $\mathrm{A}$, monensin and retro-2 and assessed inflammatory mediator release post-LPS stimulation. Our data showed that inhibitor treatment and Sec $22 \mathrm{~b}$ knockdown did not have a cumulative effect on NO, TNF and IL-6 secretion (Figure 6D-F), thereby implying that $\operatorname{Sec} 22 \mathrm{~b}$ is largely responsible for this phenotype.

\section{Sec22b participates in the nuclear translocation of NF- $\mathrm{KB}$}

To understand how Sec22b regulates LPSinduced responses, we evaluated the impact of Sec22b knockdown on LPS-induced signal transduction cascades leading to the expression of iNOS. Apart from an impairment of JNK phosphorylation, silencing of Sec22b in JAWS-II DC had no significant effect on LPS-induced signaling events and on NF- $\kappa \mathrm{B}$ expression (Figure 7A). To determine whether Sec22b is involved in the nuclear translocation of NF- $\kappa B$, we stimulated JAWS-II cells expressing either a shScr or a shSec22b with LPS and quantified
NF- $\kappa$ B nuclear translocation using imaging flow cy tom etry (Figure 7 B - C ) and immunoflurescence confocal microscopy (Figure 7D-E). Our results show that knockdown of Sec22b hampered LPS-induced $\mathrm{NF}-\kappa \mathrm{B}$ nuclear translocation. We made a similar observation in LPS-stimulated RAW264.7 macrophages treated with siRNA to Sec22b (Figure 7F-G). These data indicate that the secretory pathway-resident SNARE Sec22b modulates proinflammatory effector production by participating to the nuclear translocation of NF- $\kappa \mathrm{B}$.

Since Sec22b is present in the ER-ERGIC-Golgi circuitry $(8,29)$, we hypothesized that NF- $\kappa B$ and Sec $22 \mathrm{~b}$ are found in the same compartments. To test this, we used immunofluorescence to examine the colocalization of NF- $\kappa \mathrm{B}$ with Sec22b, as well as other ERGIC and Golgi proteins. In control and LPS-stimulated BMDC, we found partial colocalization under steady state conditions, which disappeared following LPS-induced migration of NF- $\kappa \mathrm{B}$ to the nucleus (Figure 8A-B and Figures S2 \& S3). We also observed that Sec22b was present in the nucleus; LPS slightly, but significantly, increased the proportion of $\mathrm{Sec} 22 \mathrm{~b}$ in the nucleus compared to the entire cell (Figure 8C). Similarly, immunogold electron microscopy revealed that NF- $\mathrm{kB}$ and Sec $22 \mathrm{~b}$ cooccur in membranous structures in the cytoplasm, and increasingly in the nucleus after LPS stimulation (Figure 8D and Figure S3). Both $\mathrm{NF}-\kappa \mathrm{B}$ and Sec $22 \mathrm{~b}$ were found in nuclear regions that do not co-stain with DAPI. Since nucleoli also exhibit this characteristic (30), we asked whether Sec22b colocalizes with nucleolar marker fibrillarin (31), and found that Sec22b was not found in these structures (Figure 8E-F and Videos S1 \& S2). Collectively, these findings revealed that NF- $\kappa B$ is an ERGolgi-associated protein that necessitates Sec22b to translocate to the nucleus.

\section{Discussion}

In dendritic cells, microbial-derived molecules induce the expression of genes encoding proinflammatory mediators and anti-microbial molecules through complex signalling cascades that culminate in the activation of specific transcription factors (32). In the present study, 
we aimed at further elucidating the mechanisms and pathways associated to the production of NO and inflammatory mediators in BMDC exposed to LPS. Our main finding is that the secretory pathway, through the action of the SNARE Sec22b, plays a central role in LPS-induced responses by regulating the nuclear translocation of the transcription factor $\mathrm{NF}-\kappa \mathrm{B}$.

Whereas the signaling pathways leading to the expression of iNOS and the roles of NO have been explored in great details in various immune cell types $(16,18)$, much less is known on the sub-cellular localization of iNOS. Using electron microscopy and biochemical approaches, Vodovotz and colleagues reported that over $40 \%$ of the iNOS activity in whole sonicates was particulate in LPS-activated primary macrophages (33). They also observed that iNOS was associated to 50 to $80 \mathrm{~nm}$ vesicles of undefined nature, which did not correspond to endosomes, lysosomes or peroxysomes. Interestingly, the most intense iNOS labeling observed by immuno-electron microscopy was perinuclear, on the trans side of the trans-Golgi network (33). Upon phagocytosis, iNOS was found to associate to phagosomes through an actin-dependent mechanism (34). Our results provide evidence that in dendritic cells stimulated with LPS, iNOS is present within the secretory pathway, as it colocalizes to various extents with proteins found in the ER, the ERGIC and the Golgi. Moreover, we observed that on phagosomes, iNOS colocalized with ER-Golgi SNAREs Sec22b and Stx5, raising the possibility that the previously observed iNOS-positive vesicular structures originate from these organelles.

Given the large number of genes controlled by $N F-\kappa B$, this ubiquitous transcription factor plays a central role in the regulation of inflammation, and innate and adaptive immune responses, including LPS-induced responses in dendritic cells $(32,35)$. In resting cells, NF- $\kappa B$ dimers are sequestrated in an inactive state in the cytoplasm by a member of the inhibitory proteins known as I $\kappa$ Bs (36), preventing NF- $\kappa B$ from being recognized by the nuclear import machinery (37). Cell stimulation triggers signaling cascades leading to the phosphorylation of IкB, its ubiquitination, and degradation by the proteasome $(38,39)$,

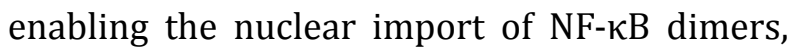
their binding to consensus DNA sequences, and the activation of target gene expression (25). $\mathrm{Nucleocytoplasmict} \mathrm{trafficking} \mathrm{of}$ macromolecules such as transcription factors is tightly controlled by importins and exportins, through the recognition of nuclear localization signals and nuclear export signals (40). Using pharmacological inhibitors, we obtained evidence that the ER-Golgi circuitry contributes to optimal LPS-induced responses in BMDC, through the regulation of MAPK phosphorylation and NF- $\kappa \mathrm{B}$ nuclear translocation. The fact that Erk1/2 and p38 are involved in LPS-induced NO release (41) led us to the finding that secretory pathway blockade inhibited Erk1/2 and p38 phosphorylation, which may explain in part why NO secretion decreases. Consistent with our findings, previous studies with RAW264.7 macrophages and with primary mouse astrocytes revealed that ER stress induced by either tunicamycin or brefeldin A inhibited LPS-induced iNOS expression $(42,43)$. These findings highlight a previously unappreciated role for the ER-Golgi secretory pathway in the translocation of NF- $\kappa B$ to the nucleus.

Through their role in membrane fusion, the ER/ ERGIC-resident SNAREs Sec22b and Stx5 participate in various cellular processes including secretory autophagy (28), embryonic development (15), and plasma membrane expansion (44). These two SNAREs also play a key role in phagosome maturation and function by regulating the delivery of ER and ERGIC resident proteins to phagosomes in macrophages and dendritic cells. Remarkably, we found that Sec22b was needed for the expression, at the mRNA and protein levels, of iNOS, TNF and IL-6. In immune cells, LPSinduced responses are largely dependent on $\mathrm{NF}-\kappa \mathrm{B}$, as it regulates the expression of several genes including iNOS, IL-6, and TNF (25). Although Sec22b KD also decreased MAPK phosphorylation, its effect on NF- $\kappa B$ translocation was surprising but not without precedent. For one, ER- and Golgi-resident proteins such as HSP90 and golgin-97 have been implicated in the translocation of NF- $\kappa \mathrm{B}$ to the nucleus $(45,46)$. Since we observed that NF$\kappa \mathrm{B}$ was present in the ERGIC and Golgi, it is reasonable to postulate that $\operatorname{Sec} 22 \mathrm{~b}$ plays an active role in the cytoplasmic-nuclear shuttling 
of NF- $\kappa B$. Second, SNAREs and their chaperones are required for nuclear pore assembly and sealing (47). Our finding that Sec22b and NF- $\kappa B$ co-occured in the cytoplasm and nucleus further supports the notion that Sec22b is part of the circuity that, with help from importins $(37,40)$, shepherds NF- $\kappa B$ translocation.

There is growing evidence that SNAREs and alternatively spliced isoforms access the nucleus where they perform unsuspected functions (47-52). In a study aimed at determining the subcellular localization of Stx17, it was found that this SNARE is present in both the cytoplasm and nucleus of several cell types (52). The absence of homology with known nuclear localization signal (NLS) consensus sequences led the authors to speculate about the existence of a yet uncharacterized nuclear transport mechanism for Stx17. The meaning of the presence of Stx17 in the nucleus remains to be elucidated. Similarly, the SNARE SNAP-47, was found in the ER, ERGIC and nucleus of HeLa cells (50). Further analyses revealed the presence of several putative NLS and two nuclear export signals in SNAP-47. Treatment of HeLa cells with leptomycin B, which inhibits CRM-1/ exportin1-dependent nuclear export led to nuclear accumulation of SNAP-47, suggesting a functional nuclear export signal in SNAP-47 (50). SNAP-47 was previously shown to interact with Stx3 (49). Interestingly, a splice variant of Stx 3 was found to undergo nuclear translocation, to bind to several transcription factors, and act as a transcriptional activator (48). An isoform of Stx1, lacking a transmenbrane domain, was also found to localize in the nucleus (51). The observation that Sec22b was present in non-nucleolar compartments of the nucleus raises the interesting possibility that Sec22b partakes in nuclear dynamics and gene regulation. Since the ' $a$ ', 'b' and 'c' isoforms of Sec22 possess transmembrane domains (29), we do not exclude the possibility that Sec $22 \mathrm{~b}$ undergoes cleavage prior to its nuclear translocation. Hence, how Sec22b migrates to the nucleus is subject of future research.

In sum, our findings demonstrated that the SNARE Sec22b controls inflammatory effector release by facilitating the translocation of NF$\kappa \mathrm{B}$ from the secretory pathway to the nucleus.

\section{Experimental procedures}

\section{Ethics statement}

Mouse work was done as stipulated by protocol 1706-06, which was approved by the Comité Institutionel de Protection des Animaux of the INRS-Centre Armand-Frappier Santé Biotechnologie. This protocol respects procedures on animal practice promulgated by the Canadian Council on Animal Care, described in the Guide to the Care and Use of Experimental Animals.

\section{Antibodies and inhibitors}

Rabbit and mouse antibodies anti-iNOS, -SAPK/ JNK, -p-SAPK/JNK (Thr183/Tyr185), -p38, -pp38 (Thr180/182), -p44/42 MAPK, -p-p44/42

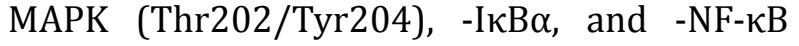
were purchased from Cell Signaling [cat. nos. 13120, 2982, 9252, 9251, 9212S, 9211, 9102, 9101, 9242, 4764, 6956]; rabbit antibodies anti-Sec22b and -Stx5 from Synaptic Systems [186003, 110053]; rabbit anti-ERGIC53/p58 and mouse anti- $\beta$-actin from Sigma [E1031, A1978]; rabbit anti-PDI and -CNX from Enzo Life Sciences [ADISPA890D, ADISPA860D]; rabbit anti-fibrillarin from Abcam [ab4566]; and rabbit anti-P115 from Proteintech [135091AP]. Pharmacological inhibitors brefeldin A (diluted to $0.5 \mu \mathrm{g} / \mathrm{ml}$ ), monensin (5 $\mu \mathrm{M})$ and retro-2 $(100 \mu \mathrm{M})$ were obtained from Molecular Probes [B7450], Sigma [M5273] and Calbiochem [554715], respectively, and were reconstituted in DMSO from Bioshop [DMS555].

\section{Cell culture}

Bone marrow-derived dendritic cells (BMDC) were differentiated from the bone marrow of 6to 8-week old female C57BL/6 mice. BMDC were differentiated for 7 days in RPMI (Life technologies) containing $10 \% \mathrm{v} / \mathrm{v}$ heatinactivated foetal bovine serum (FBS) [Life Technologies] $10 \mathrm{mM}$ HEPES (Bioshop) at $\mathrm{pH}$ 7.4, penicillin-streptomycin (Life Technologies), and $10 \% \mathrm{v} / \mathrm{v}$ X63 cell-conditioned medium as a source of granulocyte-macrophage colonystimulating factor $(53,54)$. Experiments were carried out in complete medium containing 5\% X63 cell-conditioned medium. JAWS-II dendritic 
cell-like lines stably transduced with shRNA for Sec22b (10) were a gift from D. S. Amigorena (Institut Curie). JAWS-II cells were cultured in RPMI containing 20\% heat-inactivated FBS, 10 mM HEPES at pH 7.4, penicillin-streptomycin, $10 \%$ X63 cell-conditioned medium and $40 \mu \mathrm{g} /$ $\mathrm{ml}$ puromycin (Bioshop). The mouse macrophage cell line RAW264.7 clone D3 was cultured in DMEM containing L-glutamine (Life Technologies), 10\% heat-inactivated FBS, 10 $\mathrm{mM}$ HEPES at $\mathrm{pH}$ 7.4, and penicillinstreptomycin. For microscopy experiments, BMDC and JAWS-II cells were seeded onto polyL-lysine coated coverslips (BD Biosciences, 354085) and RAW264.7 cells onto uncoated glass coverslips (Fisher, 1254581). All mammalian cells were kept in a humidified $37^{\circ} \mathrm{C}$ incubator with $5 \% \mathrm{CO}_{2}$.

Leishmania major NIH S (MHOM/SN/74/ Seidman) clone A2 (A2WF) promastigotes were provided by W. R. McMaster (University of British Columbia) and were cultured in Leishmania medium [M199-1X (Sigma) with $10 \%$ heat-inactivated FBS, $40 \mathrm{mM}$ HEPES at $\mathrm{pH}$ 7.4, $100 \mu \mathrm{M}$ hypoxanthine, $5 \mu \mathrm{M}$ hemin, $3 \mu \mathrm{M}$ biopterin, $1 \mu \mathrm{M}$ biotin, and penicillinstreptomycin] in a $26^{\circ} \mathrm{C}$ incubator. Freshly differentiated promastigotes in late stationary phase (5-day cultures at $>50 \times 10^{6}$ promastigotes $/ \mathrm{ml}$ ) were used for infections.

\section{siRNA knockdown}

RAW264.7 macrophages in the second passage were seeded onto 24-well plates and reversetransfected with Lipofectamine RNAiMAX (Thermo Fisher Scientific, 13778075) as per the manufacturer's recommendations. Cells were transfected with siRNAs targeting a scrambled sequence, Sec22b (Thermo Fisher Scientific D0018101005 and 4390815) or Stx5 (Dharmacon, L063346010005) at a final concentration of $25 \mathrm{nM}$ in a $600 \mu \mathrm{l}$ volume of complete DMEM without antibiotics for $48 \mathrm{~h}$ (55). Prior to experiments, macrophages were replenished with complete medium for $4 \mathrm{~h}$ before lipopolysaccharide (LPS) [diluted to 100 ng/ml; Sigma, L3129] stimulation. Cells were subsequently prepared for microscopy, and their culture supernatants used for NO measurements.

\section{Phagocytosis assays}

Cells were incubated at $4^{\circ} \mathrm{C}$ for 5 min with mouse serum-opsonized zymosan (Sigma, Z4250) or L. major promastigotes, followed by a 2 min spin at $298.2 \mathrm{~g}$ in a Sorvall RT7 centrifuge (56). Particle internalization was triggering by transferring cells to $37^{\circ} \mathrm{C}$, and after $15 \mathrm{~min}$ (zymosan) or $2 \mathrm{~h}$ (promastigotes), excess particles were washed thrice with warm medium. Cells were then lysed or prepared for confocal microscopy (56).

\section{NO and cytokine release measurements}

Cells were pretreated for $3 \mathrm{~h}$ with DMSO or the aforementioned chemical inhibitors and subsequently stimulated - in the presence or absence of inhibitors - with LPS, peptidoglycan (PGN) [40 $\mu \mathrm{g} / \mathrm{ml}$; Sigma, 77140], Imiquimod [12.5 $\mu \mathrm{g} / \mathrm{ml}$; Invivogen, tlrl-imq], CpG [4 $\mu \mathrm{g} / \mathrm{ml}$; Invivogen, tlrl-1585], zymosan or L. major promastigotes. Cell culture supernatants were spun to remove debris, and nitrite concentrations were assayed via the Griess reaction (57). ELISA kits were used to quantify secreted TNF (Ready-SET-Go! Mouse TNF $\alpha$ Kit, eBiosciences,88732488) and IL-6 (BD OptiEIA, Becton-Dickinson Biosciences, 555240), as per the manufacturers' instructions.

\section{Immunofluorescence confocal microscopy and imaging flow cytometry}

Cells on coverslips were fixed with $2 \%$ paraformaldehyde (Thermo Scientific, 28906) for $20 \mathrm{~min}$ and blocked and permeabilized for 17 min with a solution of $0.1 \%$ Triton X-100, $1 \%$ BSA, $6 \%$ non-fat milk, $2 \%$ goat serum, and $50 \%$ FBS. This was followed by a $2 \mathrm{~h}$ incubation with primary antibodies diluted in PBS. Then, cells were incubated for $35 \mathrm{~min}$ in a solution containing suitable combinations of AlexaFluorlinked secondary antibodies (diluted 1:500; Molecular Probes A11008, A11011, A11031, A11001, A21247) and DAPI (1:40000; Molecular Probes, D3571). $\mathrm{G}_{\mathrm{M} 1^{+}}$lipid rafts were stained with the cholera toxin-subunit BAlexaFluor 647 conjugate (1:5000; Molecular Probes, C34778) (58). Coverslips were washed three times with PBS after every step. Coverslips were mounted onto glass slides (Fisher) with Fluoromount-G (Southern 
Biotechnology Associates, 010001) and sealed with nail hardener (Sally Hansen, 45077). Cells were imaged with the $63 \mathrm{X}$ objective of an LSM780 confocal microscope (Carl Zeiss Microimaging). Control stainings revealed no cross-reactivity or background (Figure S3). Images were taken in sequential scanning mode via the ZEN 2012 software and mounted with Photoshop (Adobe). Images were analyzed using Icy Software (Institut Pasteur) (59). Colocalization (60) was evaluated using the Pearson R coefficient calculated from regions of interested defined by active contours (61) on the cytoplasm (DIC channel) or on the nucleus (DAPI). Nuclear translocation was evaluated by dividing the mean fluorescence intensity of an entire cell by that of its nucleus. Recruitment was evaluated by scoring the presence of a given protein on the phagosome membrane $(58,62)$.

Imaging flow cytometry experiments were performed as previously described (63). Briefly, cells were washed with cold PBS containing 1\% horse serum (Sigma), $0.1 \% \mathrm{NaN}_{3}$, and $5 \mathrm{mM}$ EDTA, fixed, permeabilized and stained. DNA was stained with DAPI immediately prior to running the samples through an ImageStreamX MKII flow cytometer running IDEAS software (Amnis). Nuclear translocation of NF- $\kappa B$ was evaluated using a score that quantifies the correlation of NF- $\mathrm{KB}$ and DAPI pixels on a per cell basis; a score of $>1$ indicated nuclear translocation (63).

\section{Immunogold labeling and electron microscopy}

LPS-treated treated or control BMDCs were fixed overnight at $4^{\circ} \mathrm{C}$ in $0.1 \%$ glutaraldehyde + $4 \%$ paraformaldehyde in a cocodylate buffer at $\mathrm{pH}$ 7.2. After washing, cells were treated with $1.3 \%$ osmium tetroxide in collidine buffer and dehydrated (56). Pelleted cells were embedded (SPURR, TedPella) and placed in BEEM capsules (Pelco Int, 130). After resin polymerization, samples were sectioned using an ultramicrotome (LKB Broom - 2128, Ultratome). Sections were placed on nickel grids, treated with sodium metaperiodate and blocked with $1 \%$ BSA in PBS1X. Grids were then incubated with primary antibodies for $90 \mathrm{~min}$ (diluted 1:30 with 0.1\% BSA in PBS1X), washed, and incubated in suitable $10 \mathrm{~nm}$ (anti-mouse) or $20 \mathrm{~nm}$ (anti-rabbit) gold particle-conjugated secondary antibodies (Abcam ab39619 and ab27237) for 60 min (diluted 1:10 with $0.1 \%$ BSA in PBS1X). Washed samples were treated with uranyl acetate and lead citrate for contrast, and imaged via a Hitachi 7100 electron microscope mounted with an AMT XR-111 camera. Control stainings revealed no background or non-specificity (Figure S3).

\section{Electrophoresis and Western blotting}

Cells were washed with cold PBS containing 1 $\mathrm{mM} \mathrm{Na} \mathrm{VO}_{4}$ (Sigma) and lysed in a solution containing 1\% NP-40, 50 mM Tris-HCl (pH 7.5), $150 \mathrm{mM} \mathrm{NaCl}, 1 \mathrm{mM}$ EDTA (pH 8), $1.5 \mathrm{mM}$ EGTA, $1 \mathrm{mM} \mathrm{Na} 3 \mathrm{VO}_{4}, 50 \mathrm{mM} \mathrm{NaF}, 10 \mathrm{mM}$ $\mathrm{Na}_{4} \mathrm{P}_{2} \mathrm{O}_{7}$, and complete protease inhibitors (Roche) (56). After incubation at $-70^{\circ} \mathrm{C}$, lysates were centrifuged for $15 \mathrm{~min}$ to remove insoluble material. After protein quantification, $30 \mu \mathrm{g}$ of sample was boiled $\left(100^{\circ} \mathrm{C}\right)$ for $6 \mathrm{~min}$ in SDS sample buffer and migrated in SDS-PAGE gels. Proteins were transferred onto HybondECL membranes (Amersham Biosciences, 10600003), blocked for $2 \mathrm{~h}$ in TBS1X-0.1\% Tween containing 5\% BSA, incubated with primary antibodies (diluted in TBS1X-0.1\% Tween containing 5\% BSA) overnight at $4{ }^{\circ} \mathrm{C}$, and thence with suitable HRP-conjugated secondary antibodies (GE Healthcare NA931V and NA934V) for $1 \mathrm{~h}$ at room temperature. Washed membranes were incubated in ECL (GE Healthcare, RPN2106) and immunodetection was achieved via chemiluminescence (62). Densitometric analysis of Western blot bands was done using the AlphaEase FC software (Alpha Innotech) with $\beta$-actin used as loading control.

\section{RNA extractions and RT-qPCR}

RNA from BMDC and JAWS-II cells was extracted using TRIzol (Life Technologies, 15596026) as per the manufacturer's protocol. Purified RNA was reverse-transcribed (64) and cDNA was submitted to RT-qPCR analysis using the iTaq Universal SYBR Green Supermix (BioRad, 1725121). Samples were amplified in duplicate on a MX3000P instrument (Stratagene), and the following primers were 
u s e d

I $n$ o s :

CTGCTGGTGGTGACAAGCACATTT-3' (AD529F) a n d ATGTCATGAGCAAAGGCGCAGAAC-3' (AD530R); $T n f$ :

5

GACGTGGAAGTGGCAGAAGAG-3' (AD537F) and 5'-TGCCACAAGCAGGAATGAGA-3' (AD538R); Il6: 5'-ACAACCACGGCCTTCCCTACTT-3' (AD539F) a $n$ d 5 ' CACGATTTCCCAGAGAACATGTG-3' (AD540R); a $\mathrm{n} \mathrm{d}$ H p rt:

GTTGGATACAGGCCAGACTTTGTTG-3' (AD55F) a $\mathrm{n} d$ 5 ' GATTCAACTTGCGCTCATCTTAGGC-3' (AD56R). MxPro-MX2000P software (Stratagene) was used to record cycle threshold $\left(\mathrm{C}_{T}\right)$ values, which were analyzed by the $2-\Delta \Delta C T$ method. Data were normalized to Hprt and expressed in terms of fold change differences relative to nonstimulated cells.

\section{Data analysis}

Statistical differences between two or multiple groups were assessed via the Mann-Whitney $U$ test or one/two-way ANOVA followed by Bonferroni post-hoc tests, respectively. Twoway ANOVA was used to evaluate timedependent differences in inflammatory mediator release. Differences were considered significant when $p<0.05$ and graphs were constructed using GraphPad Prism 5.0 (GraphPad Software Inc).

Data availability: All data are contained within the article and its supporting information. 


\section{Acknowledgements}

We are grateful to D. S. Amigorena for the kind gift of shRNA-transduced JAWS-II cells, W. R. McMaster for providing L. major promastigotes, J. Tremblay for assistance in immunofluorescence experiments, and A. Nakamura for assistance in electron microscopy.

\section{Funding and additional information}

This work was supported by Natural Science and Engineering Research Council of Canada grant 1055043 to AD and by Canadian Institutes of Health Research (CIHR) grant PJT-159647 to SS. AD is the holder of the Canada Research Chair on the Biology of intracellular parasitism. GAD was partially supported by a CIHR Frederick Banting and Charles Best Doctoral Award and by bridging funds from the Centre for Host-Parasite Interactions.

\section{Author contributions}

Conceived and designed the experiments: GAD, RD, and AD. Performed the experiments: GAD, RD, JD and AF. Analyzed the data: GAD, RD, JD, AF, SS and AD. Contributed reagents/materials/analysis tools: SS. Wrote the paper: GAD and AD. All authors discussed the findings and commented on the manuscript.

Conflict of Interest: The authors declare no conflicts of interest in regards to this manuscript.

\section{References}

1. Gordon, S. (2016) Phagocytosis: An Immunobiologic Process. Immunity 44, 463-475

2. Guermonprez, P., Saveanu, L., Kleijmeer, M., Davoust, J., Van Endert, P., and Amigorena, S. (2003) ER-phagosome fusion defines an MHC class I cross-presentation compartment in dendritic cells. Nature 425, 397-402

3. Bonifacino, J. S., and Glick, B. S. (2004) The Mechanisms of Vesicle Budding and Fusion. Cell 116, 153-166

4. Murray, R. Z., and Stow, J. L. (2014) Cytokine Secretion in Macrophages: SNAREs, Rabs and Membrane Trafficking. Frontiers in Immunology $\mathbf{5}$

5. Levin, R., Grinstein, S., and Canton, J. (2016) The life cycle of phagosomes: formation, maturation, and resolution. Immunological Reviews 273, 156-179

6. Gagnon, E., Duclos, S., Rondeau, C., Chevet, E., Cameron, P. H., Steele-Mortimer, O., Paiement, J., Bergeron, J. J., and Desjardins, M. (2002) Endoplasmic reticulum-mediated phagocytosis is a mechanism of entry into macrophages. Cell 110, 119-131

7. Murray, R. Z., Kay, J. G., Sangermani, D. G., and Stow, J. L. (2005) A role for the phagosome in cytokine secretion. Science 310, 1492-1495

8. Dingjan, I., Linders, P. T. A., Verboogen, D. R. J., Revelo, N. H., Beest, M. t., and Bogaart, G. v. d. (2018) Endosomal and Phagosomal SNAREs. Physiological Reviews 98, 1465-1492 
9. Pagan, J. K., Wylie, F. G., Joseph, S., Widberg, C., Bryant, N. J., James, D. E., and Stow, J. L. (2003) The t-SNARE syntaxin 4 is regulated during macrophage activation to function in membrane traffic and cytokine secretion. Curr Biol 13, 156-160

10. Cebrian, I., Visentin, G., Blanchard, N., Jouve, M., Bobard, A., Moita, C., Enninga, J., Moita, L. F., Amigorena, S., and Savina, A. (2011) Sec22b regulates phagosomal maturation and antigen crosspresentation by dendritic cells. Cell 147, 1355-1368

11. Nair-Gupta, P., Baccarini, A., Tung, N., Seyffer, F., Florey, O., Huang, Y., Banerjee, M., Overholtzer, M., Roche, Paul A., Tampé, R., Brown, Brian D., Amsen, D., Whiteheart, Sidney W., and Blander, J. M. (2014) TLR Signals Induce Phagosomal MHC-I Delivery from the Endosomal Recycling Compartment to Allow Cross-Presentation. Cell 158, 506-521

12. Abuaita, B. H., Burkholder, K. M., Boles, B. R., and O’Riordan, M. X. (2015) The Endoplasmic Reticulum Stress Sensor Inositol-Requiring Enzyme 1 $\alpha$ Augments Bacterial Killing through Sustained Oxidant Production. mBio 6

13. Alloatti, A., Rookhuizen, D. C., Joannas, L., Carpier, J. M., Iborra, S., Magalhaes, J. G., Yatim, N., Kozik, P., Sancho, D., Albert, M. L., and Amigorena, S. (2017) Critical role for Sec22bdependent antigen cross-presentation in antitumor immunity. $J$ Exp Med 214, 2231-2241

14. Kagan, J. C., Stein, M.-P., Pypaert, M., and Roy, C. R. (2004) Legionella Subvert the Functions of Rab1 and Sec22b to Create a Replicative Organelle. J Exp Med 199, 1201-1211

15. Wu, S.-R. J., Khoriaty, R., Kim, S. H., O’Shea, K. S., Zhu, G., Hoenerhoff, M., Zajac, C., Oravecz-Wilson, K., Toubai, T., Sun, Y., Ginsburg, D., and Reddy, P. (2019) SNARE protein SEC22B regulates early embryonic development. Scientific Reports 9, 11434

16. Xue, Q., Yan, Y., Zhang, R., and Xiong, H. (2018) Regulation of iNOS on Immune Cells and Its Role in Diseases. International Journal of Molecular Sciences 19

17. Miller, B. H., Fratti, R. A., Poschet, J. F., Timmins, G. S., Master, S. S., Burgos, M., Marletta, M. A., and Deretic, V. (2004) Mycobacteria inhibit nitric oxide synthase recruitment to phagosomes during macrophage infection. Infect Immun 72, 2872-2878

18. Bogdan, C. (2015) Nitric oxide synthase in innate and adaptive immunity: an update. Trends Immunol 36, 161-178

19. Qadoumi, M., Becker, I., Donhauser, N., Röllinghoff, M., and Bogdan, C. (2002) Expression of Inducible Nitric Oxide Synthase in Skin Lesions of Patients with American Cutaneous Leishmaniasis. Infect Immun 70, 4638-4642

20. Tzeng, E., Billiar, T. R., Robbins, P. D., Loftus, M., and Stuehr, D. J. (1995) Expression of human inducible nitric oxide synthase in a tetrahydrobiopterin (H4B)-deficient cell line: H4B promotes assembly of enzyme subunits into an active dimer. Proceedings of the National Academy of Sciences 92, 11771-11775

21. Webb, J. L., Harvey, M. W., Holden, D. W., and Evans, T. J. (2001) Macrophage Nitric Oxide Synthase Associates with Cortical Actin but Is Not Recruited to Phagosomes. Infect Immun 69, 6391

22. Berthiaume, L., Robin, J., and Alain, R. (1982) Electron microscopic study of Bluegill virus. Can. J. Microbiol. 28, 398-402 
23. Stechmann, B., Bai, S.-K., Gobbo, E., Lopez, R., Merer, G., Pinchard, S., Panigai, L., Tenza, D., Raposo, G., Beaumelle, B., Sauvaire, D., Gillet, D., Johannes, L., and Barbier, J. (2010) Inhibition of Retrograde Transport Protects Mice from Lethal Ricin Challenge. Cell 141, 231-242

24. Tartakoff, A., Vassalli, P., and Détraz, M. (1978) Comparative studies of intracellular transport of secretory proteins. J. Cell Biol. 79, 694-707

25. Liu, T., Zhang, L., Joo, D., and Sun, S.-C. (2017) NF-кB signaling in inflammation. Signal Transduction and Targeted Therapy 2, 17023

26. Aktan, F. (2004) iNOS-mediated nitric oxide production and its regulation. Life Sciences 75, 639-653

27. Jahn, R., and Scheller, R. H. (2006) SNAREs - engines for membrane fusion. Nature Reviews Molecular Cell Biology 7, 631

28. Kimura, T., Jia, J., Kumar, S., Choi, S. W., Gu, Y., Mudd, M., Dupont, N., Jiang, S., Peters, R., Farzam, F., Jain, A., Lidke, K. A., Adams, C. M., Johansen, T., and Deretic, V. (2017) Dedicated SNAREs and specialized TRIM cargo receptors mediate secretory autophagy. The EMBO journal 36, $42-60$

29. Sun, W., Tian, B.-x., Wang, S.-h., Liu, P.-j., and Wang, Y.-c. (2020) The Function of SEC22B and its Role in Human Diseases. Cytoskeleton n/a

30. Kodiha, M., Bański, P., and Stochaj, U. (2011) Computer-based fluorescence quantification: a novel approach to study nucleolar biology. BMC Cell Biology 12, 25

31. Ochs, R. L., Lischwe, M. A., Spohn, W. H., and Busch, H. (1985) Fibrillarin: a new protein of the nucleolus identified by autoimmune sera. Biology of the Cell 54, 123-133

32. Qian, C., and Cao, X. (2018) Dendritic cells in the regulation of immunity and inflammation. Seminars in Immunology 35, 3-11

33. Vodovotz, Y., Russell, D., Xie, Q. W., Bogdan, C., and Nathan, C. (1995) Vesicle membrane association of nitric oxide synthase in primary mouse macrophages. The Journal of Immunology 154, 2914

34. Miller, B. H., Fratti, R. A., Poschet, J. F., Timmins, G. S., Master, S. S., Burgos, M., Marletta, M. A., and Deretic, V. (2004) Mycobacteria Inhibit Nitric Oxide Synthase Recruitment to Phagosomes during Macrophage Infection. Infect Immun 72, 2872

35. Hofer, S., Rescigno, M., Granucci, F., Citterio, S., Francolini, M., and Ricciardi-Castagnoli, P. (2001) Differential activation of NF-אB subunits in dendritic cells in response to Gram-negative bacteria and to lipopolysaccharide. Microbes and Infection 3, 259-265

36. Ghosh, S., and Baltimore, D. (1990) Activation in vitro of NF-кB" by phosphorylation of its inhibitor IкB". Nature 344, 678-682

37. Turpin, P., Hay, R. T., and Dargemont, C. (1999) Characterization of $\mathrm{I} \kappa \mathrm{B} \alpha$ Nuclear Import Pathway. Journal of Biological Chemistry 274, 6804-6812

38. Henkel, T., Machleidt, T., Alkalay, I., Krönke, M., Ben-Neriah, Y., and Baeuerle, P. A. (1993)

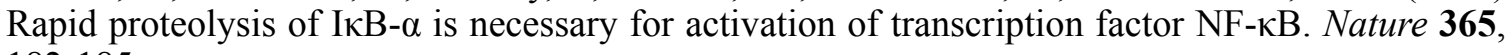
$182-185$ 
39. Kanarek, N., and Ben-Neriah, Y. (2012) Regulation of NF- $\kappa$ B by ubiquitination and degradation of the IкBs. Immunological Reviews 246, 77-94

40. Aggarwal, A., and Agrawal, D. K. (2014) Importins and Exportins Regulating Allergic Immune Responses. Mediators of Inflammation 2014, 476357

41. Feng, G.-J., Goodridge, H. S., Harnett, M. M., Wei, X.-Q., Nikolaev, A. V., Higson, A. P., and Liew, F.-Y. (1999) Extracellular Signal-Related Kinase (ERK) and p38 Mitogen-Activated Protein (MAP) Kinases Differentially Regulate the Lipopolysaccharide-Mediated Induction of Inducible Nitric Oxide Synthase and IL-12 in Macrophages: \&lt;em\&gt;Leishmania\&lt;/em\&gt; Phosphoglycans Subvert Macrophage IL-12 Production by Targeting ERK MAP Kinase. The Journal of Immunology 163, 6403

42. Hosoi, T., Noguchi, J., Takakuwa, M., Honda, M., Okuma, Y., Nomura, Y., and Ozawa, K. (2014) Inhibition of inducible nitric oxide synthase and interleukin- $1 \beta$ expression by tunicamycin in cultured glial cells exposed to lipopolysaccharide. Brain Research 1558, 11-17

43. Ho, H.-J., Huang, D.-Y., Ho, F.-M., Lee, L.-T., and Lin, W.-W. (2012) Inhibition of lipopolysaccharide-induced inducible nitric oxide synthase expression by endoplasmic reticulum stress. Cellular Signalling 24, 2166-2178

44. Petkovic, M., Jemaiel, A., Daste, F., Specht, C. G., Izeddin, I., Vorkel, D., Verbavatz, J.-M., Darzacq, X., Triller, A., Pfenninger, K. H., Tareste, D., Jackson, C. L., and Galli, T. (2014) The SNARE Sec22b has a non-fusogenic function in plasma membrane expansion. Nat Cell Biol 16, 434-444

45. Feldman, I., Feldman, G. M., Mobarak, C., Dunkelberg, J. C., and Leslie, K. K. (2007) Identification of proteins within the nuclear factor-\&\#x3ba; B transcriptional complex including estrogen receptor-\&\#x3b1. American Journal of Obstetrics \& Gynecology 196, 394.e391-394.e313

46. Hsu, R.-M., Zhong, C.-Y., Wang, C.-L., Liao, W.-C., Yang, C., Lin, S.-Y., Lin, J.-W., Cheng, H.Y., Li, P.-Y., and Yu, C.-J. (2018) Golgi tethering factor golgin-97 suppresses breast cancer cell

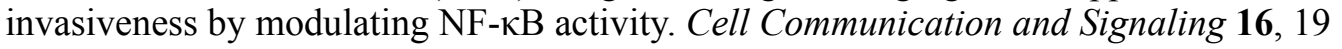

47. Baur, T., Ramadan, K., Schlundt, A., Kartenbeck, J., and Meyer, H. H. (2007) NSF- and SNAREmediated membrane fusion is required for nuclear envelope formation and completion of nuclear pore complex assembly in Xenopus laevis egg extracts. J Cell Sci 120, 2895

48. Giovannone, A. J., Winterstein, C., Bhattaram, P., Reales, E., Low, S. H., Baggs, J. E., Xu, M., Lalli, M. A., Hogenesch, J. B., and Weimbs, T. (2018) Soluble syntaxin 3 functions as a transcriptional regulator. Journal of Biological Chemistry

49. Jurado, S., Goswami, D., Zhang, Y., Molina, Alfredo J. M., Südhof, Thomas C., and Malenka, Robert C. (2013) LTP Requires a Unique Postsynaptic SNARE Fusion Machinery. Neuron 77, $542-558$

50. Kuster, A., Nola, S., Dingli, F., Vacca, B., Gauchy, C., Beaujouan, J.-C., Nunez, M., Moncion, T., Loew, D., Formstecher, E., Galli, T., and Proux-Gillardeaux, V. (2015) The Q-Soluble-NEthylmaleimide-Sensitive Factor Attachment Protein Receptor (Q-SNARE) SNAP-47 Regulates Trafficking of Selected Vesicle-Associated Membrane Proteins (VAMPs). Journal of Biological Chemistry 
51. Pereira, S., Massacrier, A., Roll, P., Vérine, A., Etienne-Grimaldi, M.-C., Poitelon, Y., RobagliaSchlupp, A., Jamali, S., Roeckel-Trevisiol, N., Royer, B., Pontarotti, P., Lévêque, C., Seagar, M., Lévy, N., Cau, P., and Szepetowski, P. (2008) Nuclear localization of a novel human syntaxin 1B isoform. Gene 423, 160-171

52. Zhang, Q., Li, J., Deavers, M., Abbruzzese, J. L., and Ho, L. (2005) The Subcellular Localization of Syntaxin 17 Varies Among Different Cell Types and Is Altered in Some Malignant Cells. Journal of Histochemistry \& Cytochemistry 53, 1371-1382

53. Ranatunga, D., Hedrich, C. M., Wang, F., McVicar, D. W., Nowak, N., Joshi, T., Feigenbaum, L., Grant, L. R., Stäger, S., and Bream, J. H. (2009) A human IL10 BAC transgene reveals tissuespecific control of IL-10 expression and alters disease outcome. Proc. Natl. Acad. Sci. U.S.A. 106, $17123-17128$

54. Hammami, A., Charpentier, T., Smans, M., and Stäger, S. (2015) IRF-5-Mediated Inflammation Limits CD8+ T Cell Expansion by Inducing HIF-1 $\alpha$ and Impairing Dendritic Cell Functions during Leishmania Infection. PLoS Pathog. 11, e1004938

55. Arango Duque, G., Fukuda, M., and Descoteaux, A. (2013) Synaptotagmin XI Regulates Phagocytosis and Cytokine Secretion in Macrophages. J Immunol 190, 1737-1745

56. Arango Duque, G., Jardim, A., Gagnon, É., Fukuda, M., and Descoteaux, A. (2019) The host cell secretory pathway mediates the export of Leishmania virulence factors out of the parasitophorous vacuole. PLoS Pathog 15, e1007982

57. Classen, A., Lloberas, J., and Celada, A. (2009) Macrophage Activation: Classical Vs. Alternative. in Macrophages and Dendritic Cells: Methods and Protocols (Reiner, N. E. ed.), Humana Press, Totowa, NJ. pp 29-43

58. Vinet, A. F., Fukuda, M., Turco, S. J., and Descoteaux, A. (2009) The Leishmania donovani lipophosphoglycan excludes the vesicular proton-ATPase from phagosomes by impairing the recruitment of synaptotagmin V. PLoS Pathog. 5, e1000628

59. de Chaumont, F., Dallongeville, S., Chenouard, N., Hervé, N., Pop, S., Provoost, T., Meas-Yedid, V., Pankajakshan, P., Lecomte, T., Le Montagner, Y., Lagache, T., Dufour, A., and Olivo-Marin, J.-C. (2012) Icy: an open bioimage informatics platform for extended reproducible research. Nature Methods 9, 690

60. Lagache, T., Sauvonnet, N., Danglot, L., and Olivo-Marin, J.-C. (2015) Statistical analysis of molecule colocalization in bioimaging. Cytometry Part $A$ 87, 568-579

61. Dufour, A., Thibeaux, R., Labruyere, E., Guillen, N., and Olivo-Marin, J. (2011) 3-D Active Meshes: Fast Discrete Deformable Models for Cell Tracking in 3-D Time-Lapse Microscopy. IEEE Transactions on Image Processing 20, 1925-1937

62. Arango Duque, G., Fukuda, M., Turco, S. J., Stäger, S., and Descoteaux, A. (2014) Leishmania Promastigotes Induce Cytokine Secretion in Macrophages through the Degradation of Synaptotagmin XI. J Immunol 193, 2363-2372

63. Fabié, A., Mai, L. T., Dagenais-Lussier, X., Hammami, A., van Grevenynghe, J., and Stäger, S. (2018) IRF-5 Promotes Cell Death in CD4 T Cells during Chronic Infection. Cell Reports 24, $1163-1175$ 
bioRxiv preprint doi: https://doi.org/10.1101/2020.09.20.305383; this version posted September 21, 2020. The copyright holder for this preprint (which was not certified by peer review) is the author/funder. All rights reserved. No reuse allowed without permission.

64. Matte, C., and Descoteaux, A. (2010) Leishmania donovani amastigotes impair gamma interferon-induced STAT $1 \alpha$ nuclear translocation by blocking the interaction between STAT1 $\alpha$ and importin- $\alpha$ 5. Infect. Immun. 78, 3736-3743 


\section{Figures}

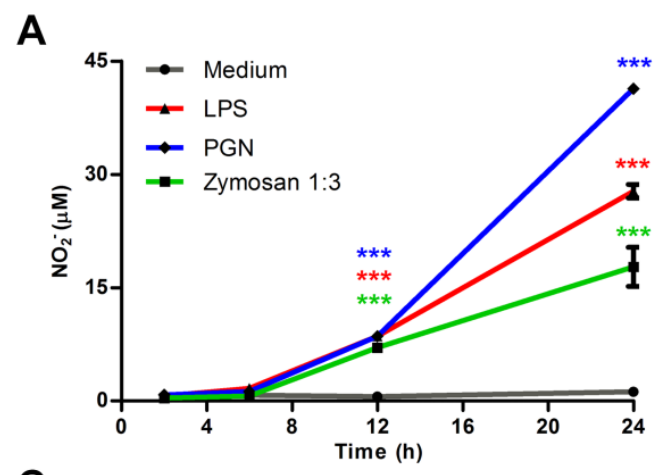

C
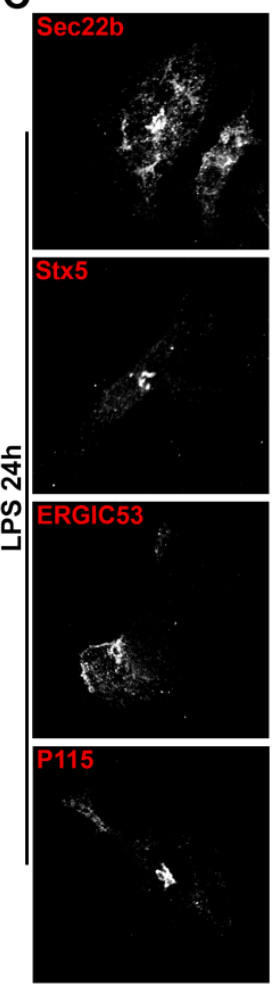
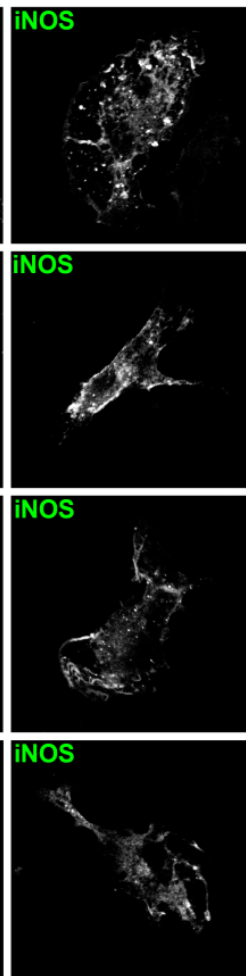

B
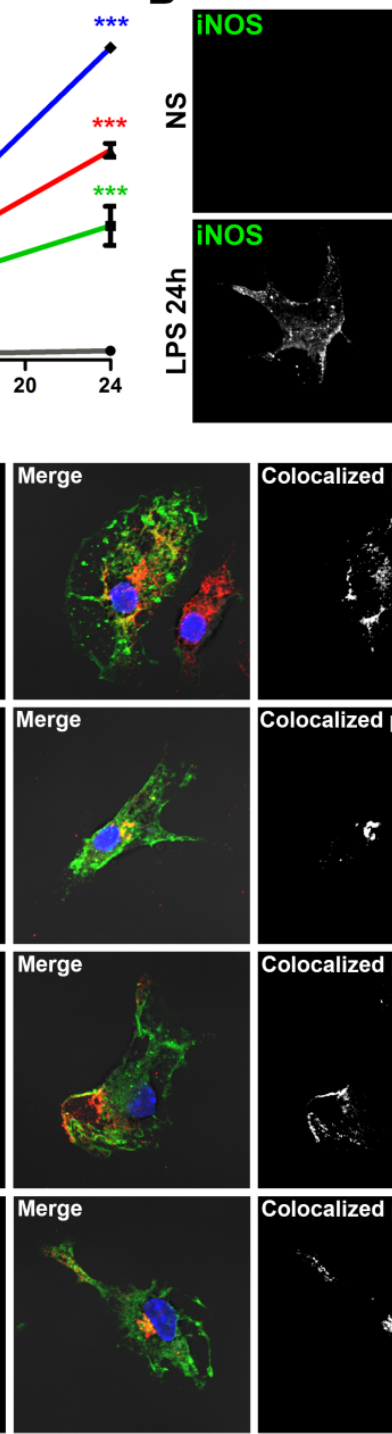
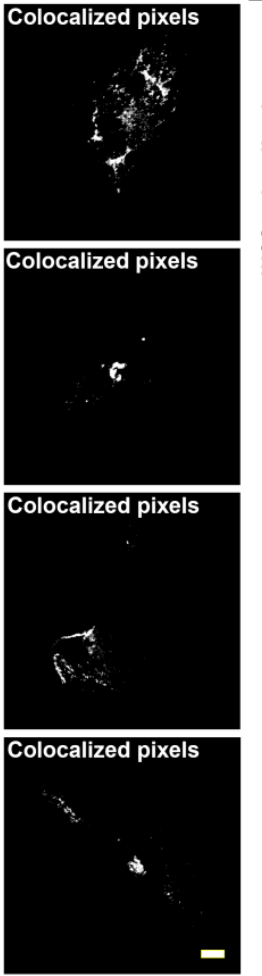

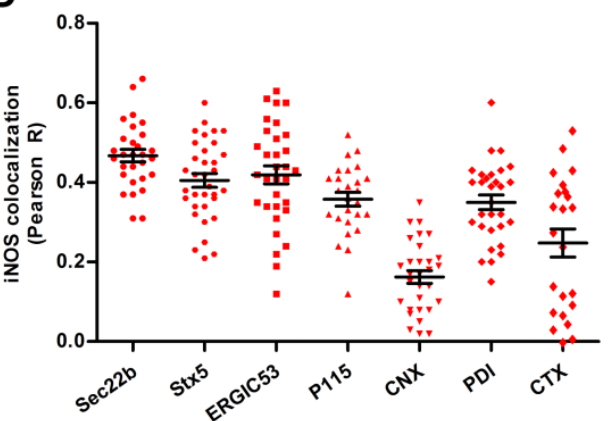

Figure 1. iNOS colocalizes with ERGIC and Golgi markers. (A) BMDC were incubated with LPS, PGN and zymosan and NO release was assessed by measuring $\mathrm{NO}_{2}$ - over a period of 2-24 h. Data are presented as mean concentration \pm SEM of three independent experiments done in triplicate wells. ***, $p<0.001$. (B) The intracellular expression of iNOS (green) in non-stimulated (NS) or LPSstimulated BMDC was visualized via confocal immunofluorescence microscopy. DNA is in blue; bar, $5 \mu \mathrm{m}$. (C) BMDC were stimulated with LPS for $24 \mathrm{~h}$ and the colocalization (white pixels, rightmost panels) of iNOS (green) with ER/ERGIC-associated proteins Sec22b, Stx5 (red); ERGIC maker ERGIC53 (red); and Golgi marker P115 (red) was assessed by immunofluorescence. DNA is in blue; bar, $5 \mu \mathrm{m}$. Images are representative of three independent experiments. (D) iNOS colocalization was quantified using the Pearson method (see also Figure S1). Data are presented as mean Pearson R coefficient \pm SEM of three independent experiments ( $\geq 10$ cells per experiment); each point represents the coefficient of a single cell. 

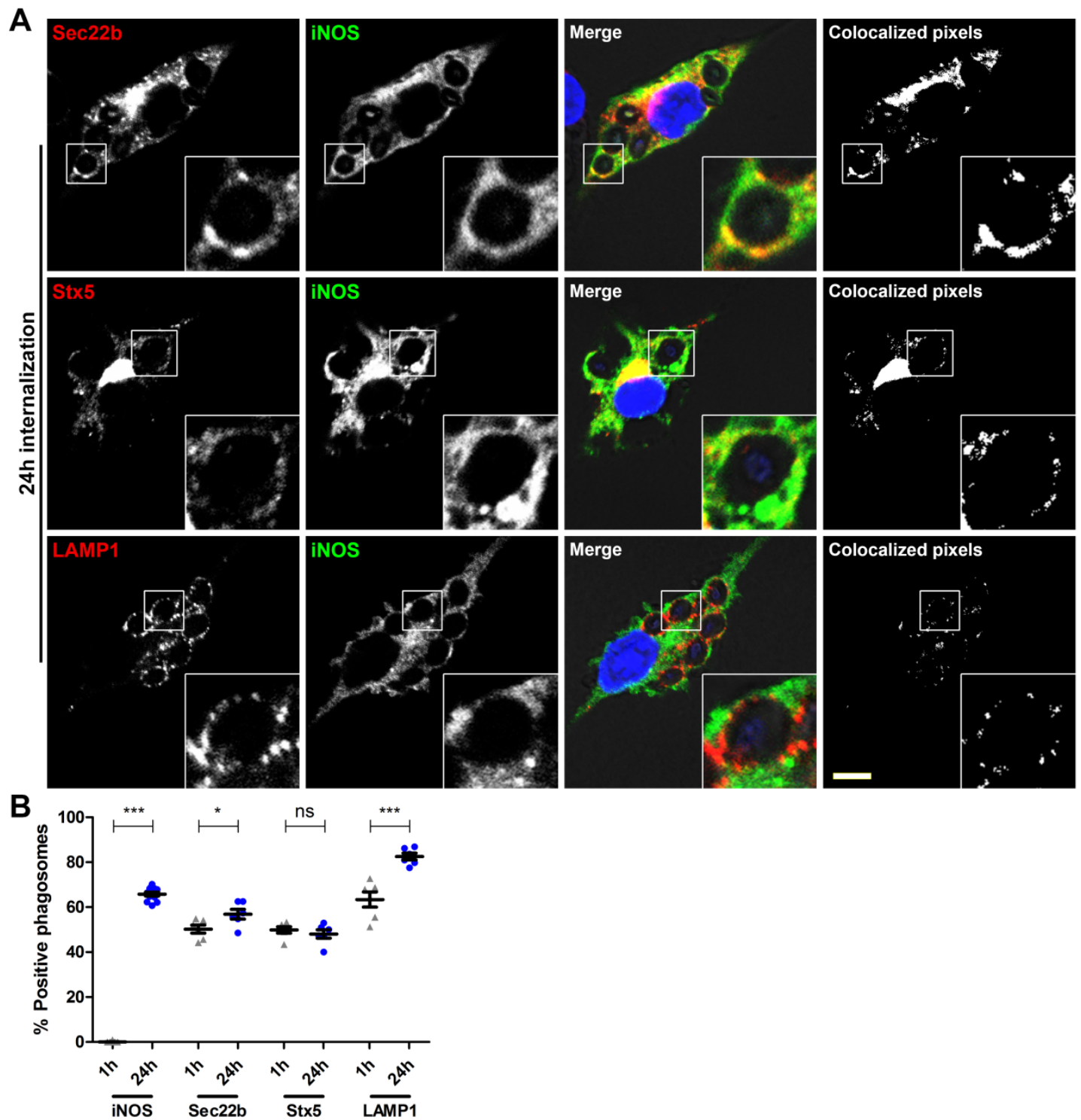

Figure 2. iNOS, Sec22b and Stx5 are recruited to the phagosome. (A) BMDC were fed with opsonised zymosan and recruitment of iNOS (green), Sec22b (red) and Stx5 (red) was visualized via immunofluorescence $24 \mathrm{~h}$ post-internalization. Recruitment of LAMP-1 (red) was used as reference. Cytoplasmic and phagosomal (3X-enlarged insets) colocalization can be observed in the rightmost panels (white pixels). DNA is in blue; bar, $5 \mu \mathrm{m}$. Images are representative of three independent experiments. (B) Phagosomal recruitment quantification of the aforementioned proteins at $1 \mathrm{~h}$ and $24 \mathrm{~h}$ post-phagocytosis. Data are presented as mean \% positive phagosomes \pm SEM of three independent experiments done in triplicate coverslips; each point represents \% recruitment in 100 phagosomes per coverslip. ${ }^{*}, p<0.05 ;{ }^{* *}, p<0.001$; ns, not significant. 


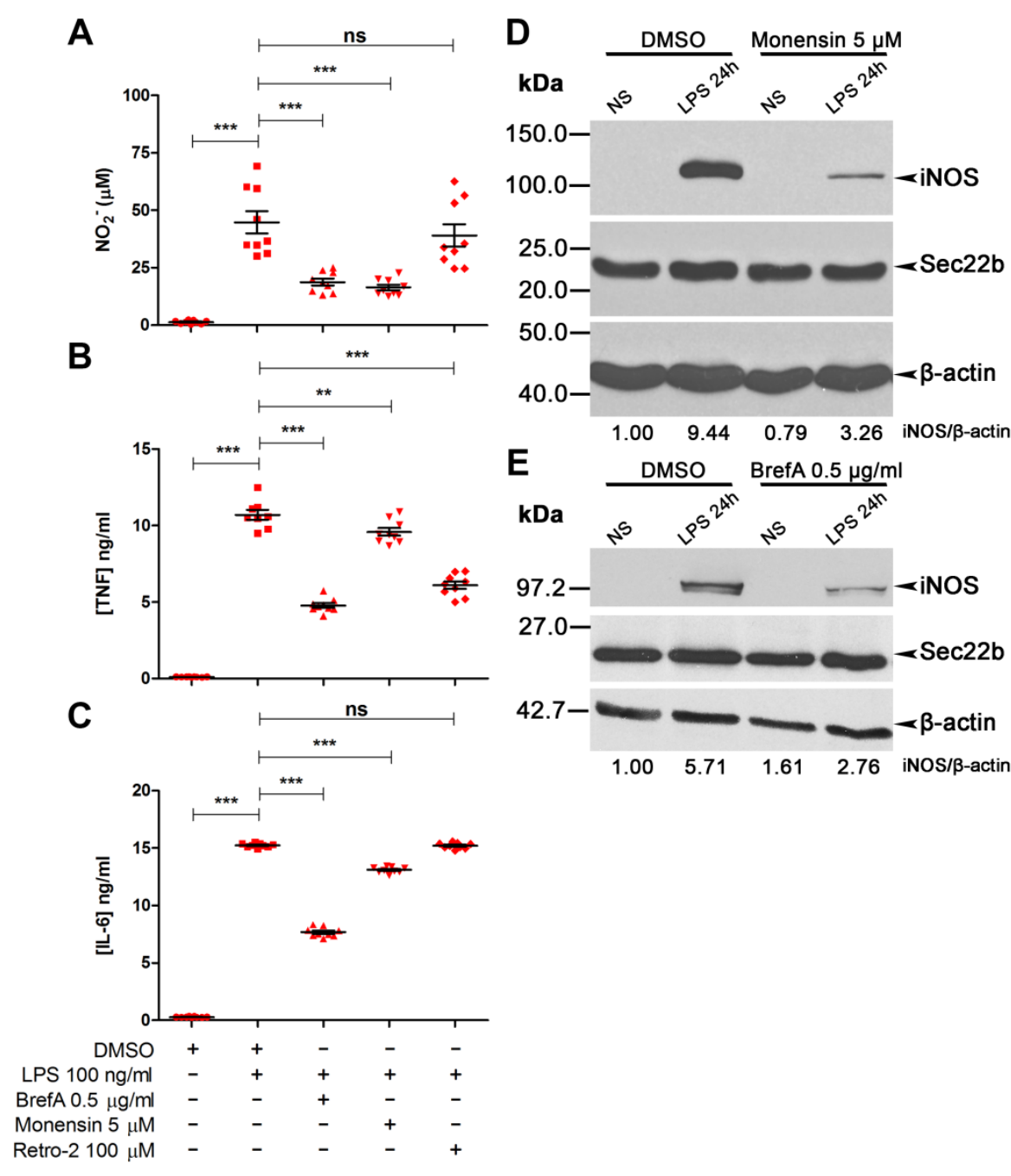

Figure 3. Inhibition of ER-Golgi trafficking blocks the production of inflammatory mediators. BMDC were incubated with DMSO or the indicated pharmacological inhibitors for $2 \mathrm{~h}$ prior to LPS stimulation for $24 \mathrm{~h}$. Cell culture supernatants were then assayed for NO (A), TNF (B) and IL-6 (C) release. Data are presented as mean concentration \pm SEM of three independent experiments done in triplicate wells; each point represents the concentration of a well. ${ }^{* *}, p<0.01$; ${ }^{* * *}, p<0.001$; ns, not significant. Immunoblotting was used to assay iNOS levels in non-stimulated (NS) or LPS-stimulated cells treated with brefeldin A (BrefA) (D) or monensin (E). iNOS densitometries were normalized with those of the $\beta$-actin loading control, and expressed relative to non-stimulated DMSO-treated cells. Images are representative of at least two experiments. 

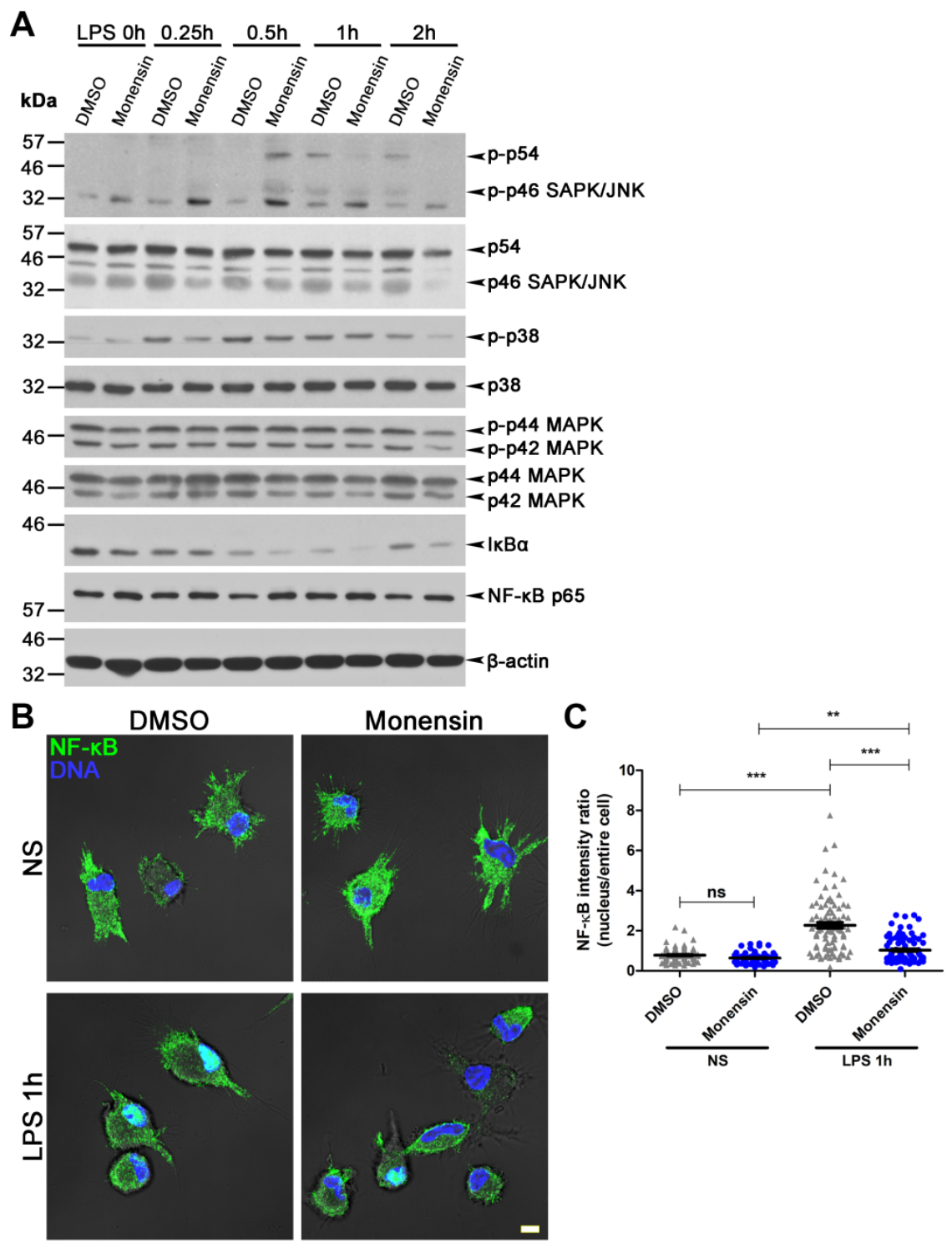

Figure 4. Inhibition of ER-Golgi trafficking inhibits MAPK phosphorylation and the translocation of NF-kB to the nucleus. (A) BMDC were incubated with DMSO or monensin for $2 \mathrm{~h}$ prior to LPS stimulation for $0.25-2 \mathrm{~h}$. Western blots show levels of total and phosphorylated MAPK, as well as I $\kappa \mathrm{B} \alpha$ and NF- $\kappa \mathrm{B} . \beta$-actin was used as loading control and images are representative of two experiments. (B) DMSO- or monensin-treated BMDC were incubated with LPS for $2 \mathrm{~h}$, and nuclear translocation of NF- $\kappa B$ (green) was visualized and quantified (C) via immunofluorescence. DNA is in blue; NS, non-stimulated; bar, $5 \mu \mathrm{m}$. Data are presented as average MFI ratios (NF- $\kappa \mathrm{B}$ MFI of nucleus/entire cell) \pm SEM of four independent experiments ( $\geq 20$ cells per experiment); each point represents the ratio of a single cell. ${ }^{* *}, p<0.01$; ${ }^{* * *}, p<0.001 ;$ ns, not significant. 

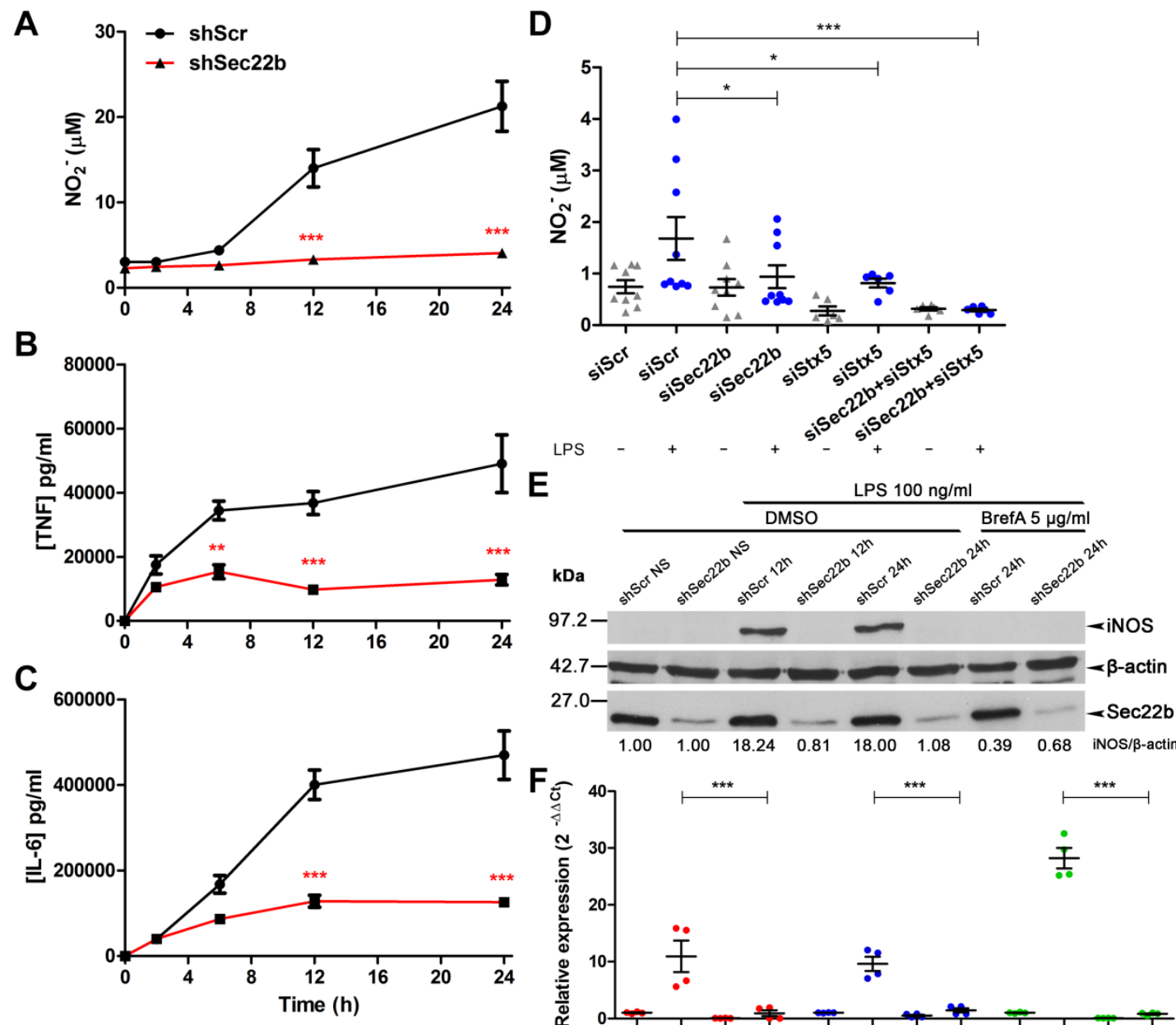

Figure 5. Sec22b is a positive regulator of inflammatory mediator release. To evaluate whether Sec22b modulates inflammatory effector secretion, LPS was used to stimulate JAWS-II cells transduced with an shRNA targeting Sec22b (shSec22b) or a scrambled sequence (shScr). Over a period of $24 \mathrm{~h}$, the release of NO (A) TNF (B) and IL-6 (C) was quantified in cell culture supernatants. Data are presented as mean concentration \pm SEM of three independent experiments done in triplicate wells. (D) NO secretion in control or LPS-treated RAW264.7 cells transfected with siRNA to a scrambled sequence, Sec $22 \mathrm{~b}$ or Stx 5 . Data are presented as mean concentration \pm SEM of at least two independent experiments done in triplicate wells; each point represents the concentration of a well. (E) iNOS protein levels in non-stimulated (NS) or LPS-stimulated Sec22bKD JAWS-II cells preincubated with DMSO or brefeldin A (BrefA) were assayed via Western blot. iNOS densitometries were normalized with those of the $\beta$-actin loading control, and expressed relative to NS-DMSO-treated cells. Images are representative of two experiments. (F) Inos, Tnf and Il6 mRNA levels in LPS-stimulated Sec22b-KD JAWS-II cells were assayed via RT-qPCR. Data are presented as mean relative expression \pm SEM of two independent experiments done in duplicate wells; points represent relative expression in each well. ${ }^{*}, p<0.05$; $^{* *}, p<0.01$; ${ }^{* *}, p<0.001$; ns, not significant. 
A
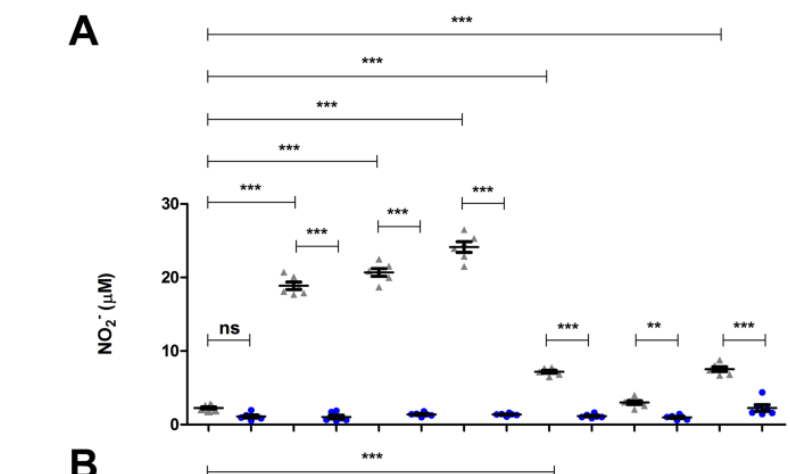

B

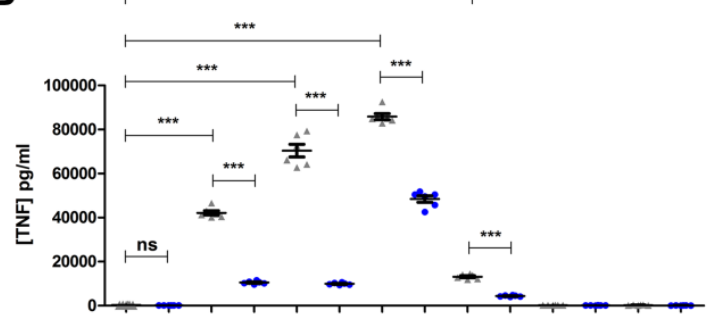

C
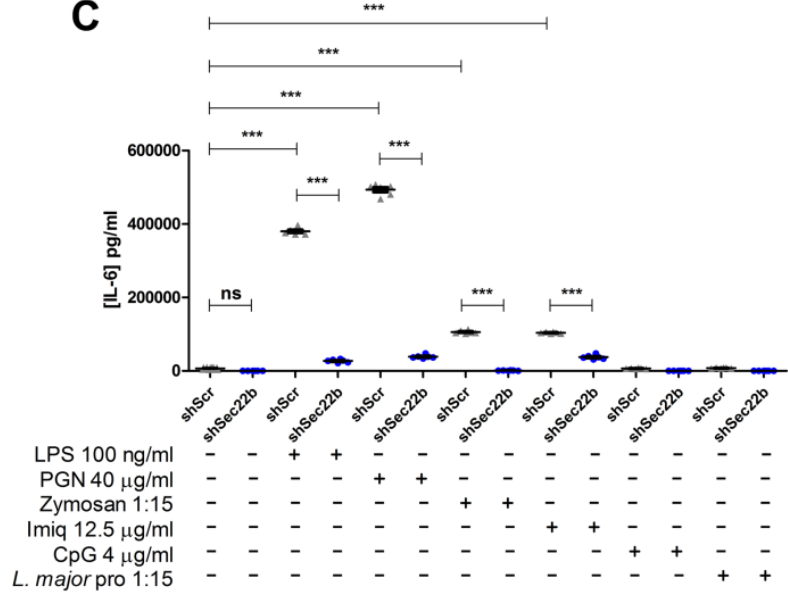

D

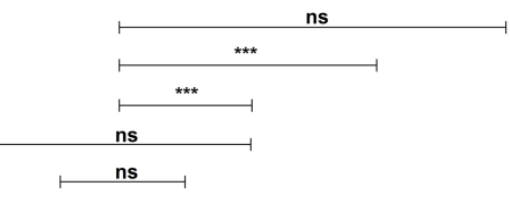

$\mathbf{E}$

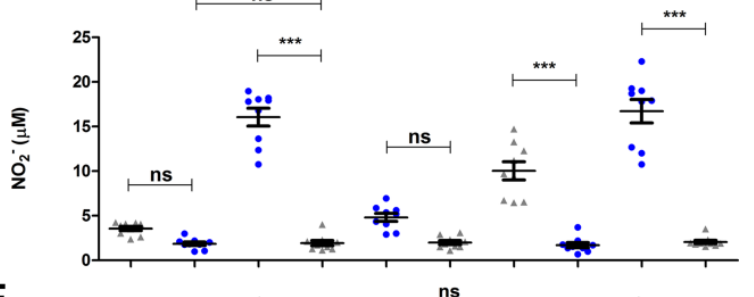

(1)

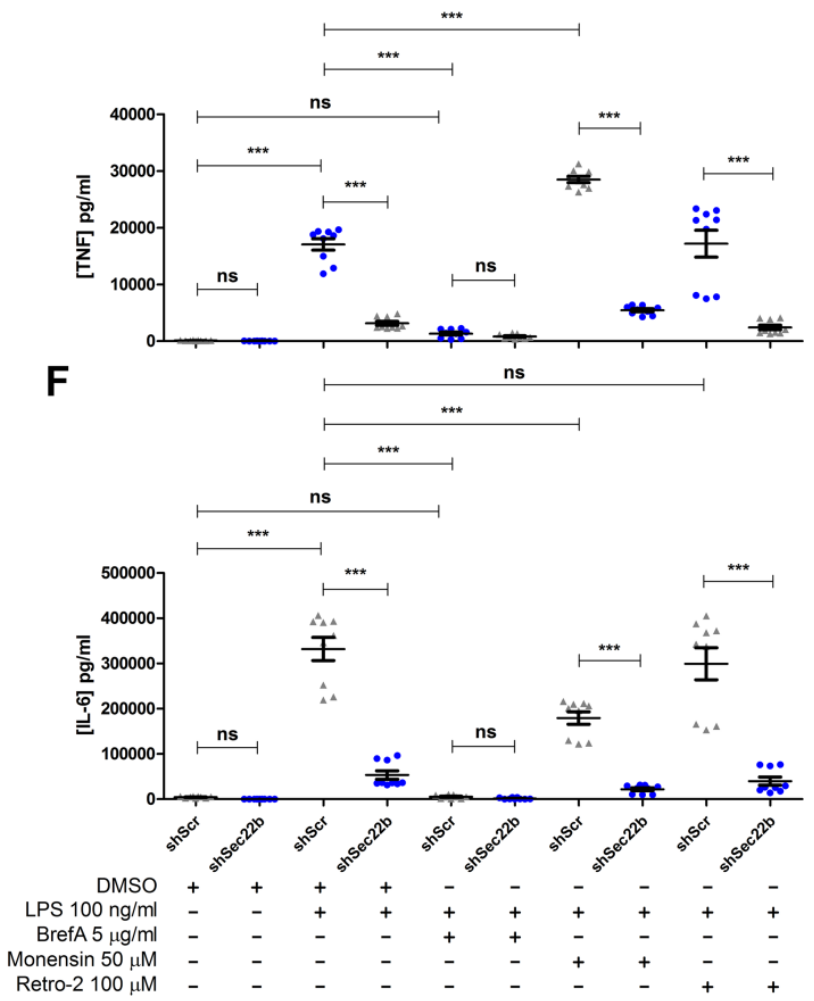

Figure 6. The role of Sec22b is neither specific to LPS stimulation, nor cumulative with chemical disruption of the secretory pathway. (A) To evaluate whether Sec $22 \mathrm{~b}$ modulates inflammatory effector secretion in an exclusively LPS-dependent manner, shScr- or shSec22btransduced JAWS-II cells were stimulated with TLR ligands LPS, PGN, zymosan, imiquimod, CpG and L. major promastigotes for $24 \mathrm{~h}$. Cell culture supernatants were then probed for the release of NO (A) TNF (B) and IL-6 (C). To show whether ER-Golgi disruption had a cumulative effect with Sec22b knockdown, JAWS-II cells were treated with pharmacological inhibitors brefeldin A (BrefA), monensin or retro-2. Cell culture supernatants were then probed for the release of NO (D) TNF (E) and IL-6 (F). In all panels, data are presented as mean concentration \pm SEM of at least two independent experiments done in triplicate wells; each point represents the concentration of a well. ${ }^{* *}, p<0.01 ;{ }^{* * *}, p<0.001 ; \mathrm{ns}$, not significant. 
A
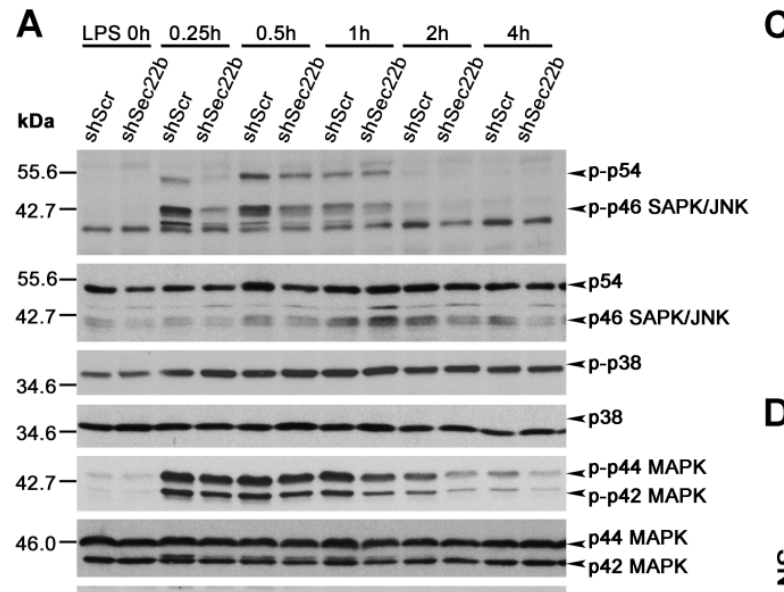

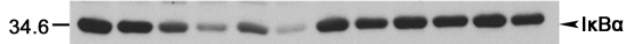

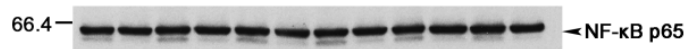
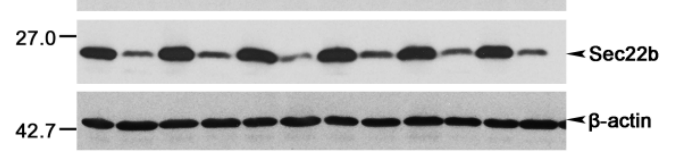

B Bright field DNA NF-kB Merge

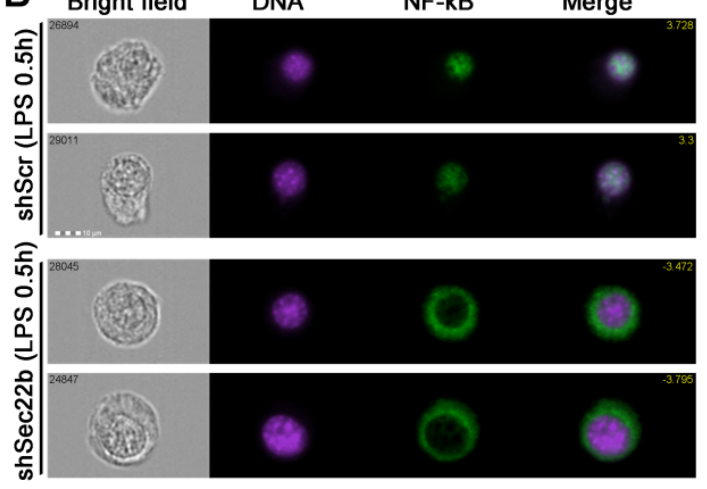

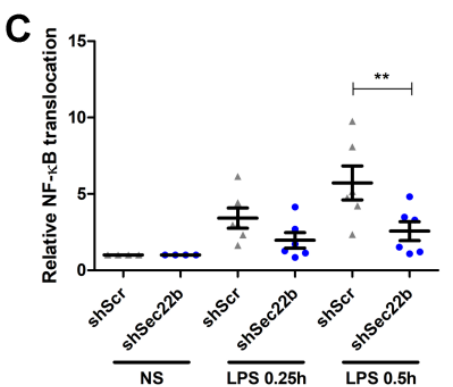
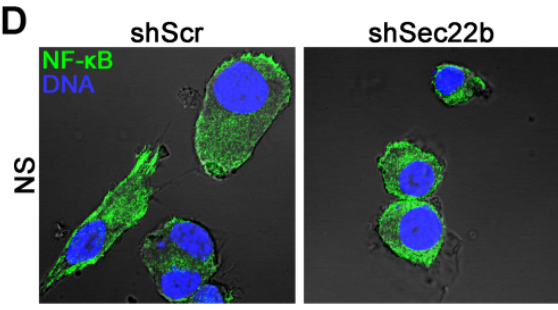

F LPS $0.5 \mathrm{~h}$
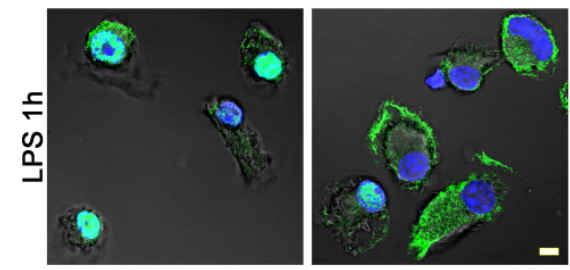

E

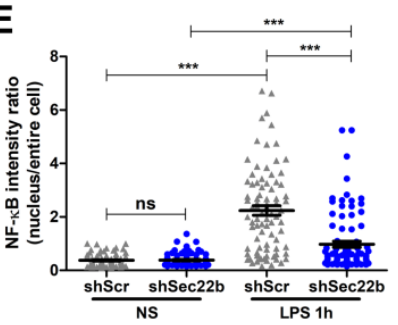

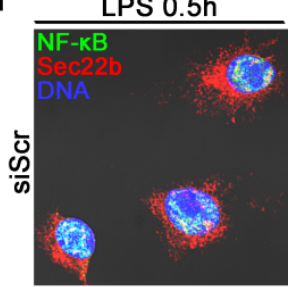

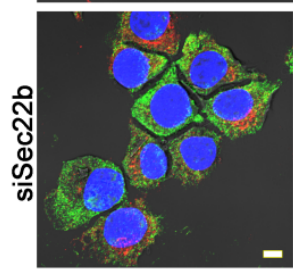

G

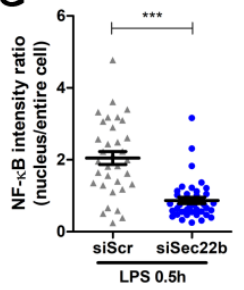

Figure 7. Sec22b modulates MAPK phosphorylation and the translocation of NF- $\mathrm{kB}$ to the nucleus. (A) ShScr- or shSec22b-transduced JAWS-II cells were stimulated with LPS for 0.25-4 h.

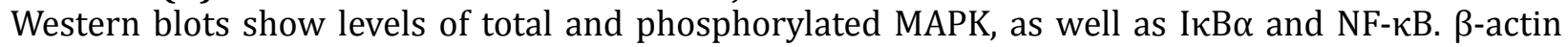
was used as loading control and images are representative of two experiments. Nuclear translocation of NF- $\kappa B$ (green) was visualized via imaging flow cytometry (B) and quantified (C). DNA is in magenta; bar, $10 \mu \mathrm{m}$. Data are presented as average NF- $\kappa B$ translocation \pm SEM relative to non-stimulated (NS) cells of at least five independent experiments; each point represents one experiment. NF- $\kappa \mathrm{B}$ translocation was also visualized and quantified via confocal microscopy in control and LPS-stimulated JAWS-II cells (D and E) or RAW264.7 macrophages (F and G). DNA is in blue; bar, $5 \mu \mathrm{m}$. Data are presented as average MFI ratios (NF- $\kappa B$ MFI of nucleus/entire cell) \pm SEM of four independent experiments ( $\geq 10$ cells per experiment); each point represents the ratio of a single cell. ${ }^{* *}, p<0.01 ;{ }^{* *}, p<0.001$; ns, not significant. 

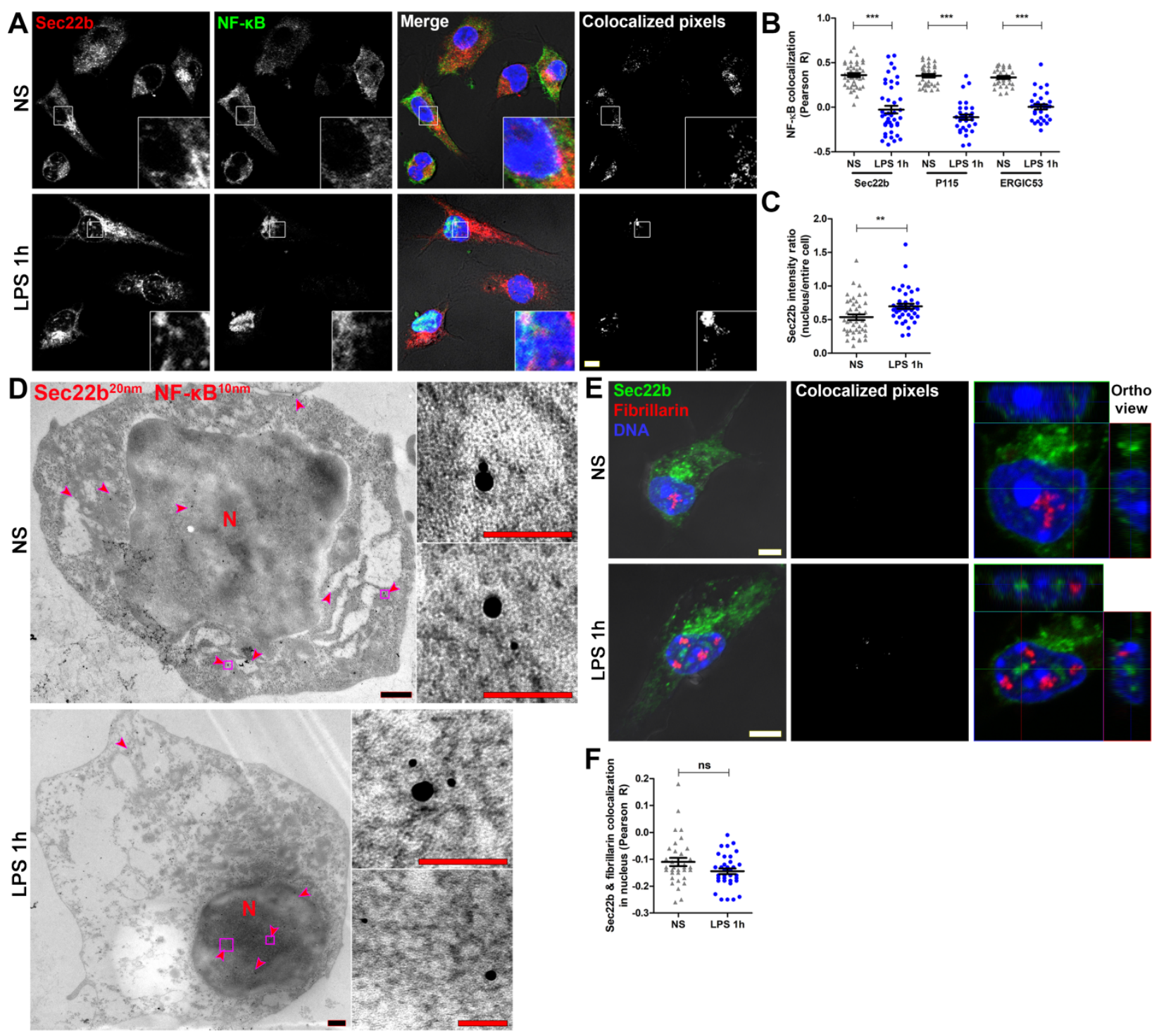

Figure 8. NF- $\mathrm{KB}$ and Sec22b co-migrate to the nucleus. (A) BMDC were stimulated with LPS for 1 $\mathrm{h}$ and the colocalization (white pixels, rightmost panels) of NF- $\kappa B$ (green) with SNARE Sec22b (red) was assessed by immunofluorescence. DNA is in blue; 3.5X-enlarged insets are shown. (B) NF- $\kappa \mathrm{B}$ colocalization was quantified using the Pearson method (see also Figure S2). (C) Quantification of Sec22b MFI ratio in nucleus relative to the entire cell. (D) Immuno-electron microscopy assessment of NF- $\mathrm{kB}$ (10 nm nanoparticles) and Sec22b (20 nm nanoparticles) expression in non-stimulated (NS) and LPS-treated BMDC. Red arrowheads indicate regions where both NF- $\kappa B$ and Sec22b nanoparticles were at a $\leq 100 \mathrm{~nm}$ distance (see also Figure S3). (E) Assessment of Sec22b (green) colocalization with nucleolar marker fibrillarin (red). Rightmost panels show orthogonal views of the nuclear region (see also Videos S1 \& S2). (F) Quantification Sec22b and fibrillarin colocalization in the nucleus. Image panels are representative of three independent experiments. Scale bars denote $5 \mu \mathrm{m}$ (white), $500 \mathrm{~nm}$ (black) and $100 \mathrm{~nm}$ (red). In (B), (C) and (F), data are presented as mean \pm SEM of three independent experiments ( $\geq 10$ cells per experiment); each point represents the measurement of a single cell. ${ }^{* *}, p<0.01 ;{ }^{* *}, p<0.001$; ns, not significant. 